was relatively low. We recommend expanding the service beyond Maccabi onto other health service organisations.

\section{ADJUSTMENT FOR MULTIPLE COMPARISONS IN A JOB AND INDUSTRY-TITLE ANALYSIS OF A CASE-CONTROL STUDY OF PROSTATE CANCER}

${ }^{1}$ Jean-François Sauvé, ${ }^{1,2}$ Jérôme Lavoué, ${ }^{3}$ Marie-Élise Parent. 'Université de Montréal, Montréal, Québec, Canada; ${ }^{2}$ INRS-Institut Armand-Frappier, Laval, Québec, Canada; ${ }^{3}$ Centre de Recherche Du CHUM, Montréal, Québec, Canada

\subsection{6/oemed-2014-102362.352}

Objectives To evaluate the impacts of empirical Bayes (EB) and semi-Bayes (SB) adjustment to account for multiple testing in a hypothesis-generating study of prostate cancer ( $\mathrm{PCa}$ ) risk by occupation and industry.

Method The study population comprises 1937 PCa cases and 1995 population controls aged $40-75$ years, all residing in Montreal. Odds ratios (OR) and 95\% confidence intervals (CI) of PCa risk for ever employment in an occupation and industry were estimated using unconditional logistic regression models adjusted for age, ancestry, and family history of PCa. EB and SB adjustment was applied to the estimates, with prior variances of $0.15,0.25$ and 0.35 selected for SB. Occupation and industry effects were considered mutually exchangeable, with the risk estimates shrunk towards their respective global mean.

Results 5 of the 89 occupations and 3 of the 63 industries had a significantly elevated $\mathrm{PCa}$ risk prior to $\mathrm{EB} / \mathrm{SB}$ adjustment, compared to an expected 2 and 1.5 categories due to random chance. The only positive association remaining significant following EB was for subjects ever employed in government $(\mathrm{OR}=1.4,95 \% \mathrm{CI} 1.1-1.5)$. The remaining elevated PCa risks with SB were found for employment in social science occupations $(\mathrm{OR}=1.5,95 \% \mathrm{CI} 1.1-2.0)$ and for forestry workers $(\mathrm{OR}=1.7,95 \%$ CI 1.1-2.6), in addition to government $(\mathrm{OR}=1.4,95 \% \mathrm{CI} 1.1-1.7)$. The choice of prior variance had a negligible impact on the estimates.

Conclusions The use of EB and SB reduced the number of positive associations compared to the unadjusted estimates. The elevated PCa risk observed for employment in government remained consistent across the adjustment approaches.

\section{SMOKING AND ALLERGIC CONTACT DERMATITIS: CAUSATION OR CORRELATION?}

${ }^{1}$ Victoria H Arrandale, ${ }^{2}$ Irena Kudla, 2,3D Linn Holness. 'Occupational Cancer Research Centre, Toronto, ON, Canada; ${ }^{2}$ St. Michael's Hospital, Toronto, ON, Canada; ${ }^{3}$ University of Toronto, Toronto, ON, Canada

\subsection{6/oemed-2014-102362.353}

Objectives Contact dermatitis (CD) is the most common occupational skin disease and includes both irritant and allergic forms (ICD and ACD). Smoking has been associated with all of $\mathrm{CD}$, hand eczema and sensitisation in previous studies, but never explored in relation to work-related ACD and ICD specifically. This abstract describes differences in patients who have a workrelated diagnosis of ICD, ACD or both ICD and ACD.

Method Data from a study of patients with possible workrelated skin or respiratory disease were used. Data included demographics, symptoms, smoking history and physician diagnoses. Differences between diagnosis groups (ICD, ACD, both) were investigated using chi square and ANOVA.

Results In total 163 subjects were diagnosed with workrelated CD. Of these, $44 \%$ were female, approximately half (51\%) were ever smokers and 30\% were atopic; the mean age was 44.9 years. ICD was diagnosed in $57 \%$ of subjects, ACD in $43 \%$ and both ICD and ACD in 14\%. Current smoking was more common among subjects with ACD (40\%) and those with both ACD and ICD (35\%) compared to those with ICD $(17 \%)(p=0.02)$; no difference in pack-years was observed.

Conclusions The rate of smoking in this sample was similar to the Canadian population. Age, sex and atopy did not differ between diagnosis groups. Current smokers were more common among those with ACD and those with ACD and ICD. The mechanism by which smoking may be related to the development of allergic skin disease remains unclear (e.g., systemic inflammation, contact, behavioural differences) but deserves further attention.

\section{AN UPDATE OF MORTALITY AND CANCER INCIDENCE AMONG ONTARIO URANIUM MINERS EXPOSED TO RADON PROGENY}

${ }^{1}$ Garthika Navaranjan, ${ }^{1,2}$ Anna Kone, 'Colin Berriault, ${ }^{1}$ Minh Do, ${ }^{1,3}$ Paul J Villeneuve, ${ }^{2}$ Loraine Marrett, 'Paul A Demers. 'Occupational Cancer Research Centre, Toronto, Ontario, Canada; ${ }^{2}$ Cancer Care Ontario, Toronto, Ontario, Canada; ${ }^{3}$ Carleton University, Ottawa, Ontario, Canada

\subsection{6/oemed-2014-102362.354}

Objectives Underground uranium mining and milling was conducted in Northern Ontario from 1955 to 1996. The Ontario uranium miner's cohort was created to study the health effects of radon and other occupational exposures. Study objectives include providing updated estimates of cancer incidence and mortality for miners exposed to radon daughters, a project funded by the Canadian Nuclear Safety Commission.

Method The cohort of mine and mill workers was created using data from the National Dose Registry (Canada's ionising radiation exposure registry), and the Ontario Mining Master File (containing work history information collected during annual chest x-rays) data. The cohort consists of men who worked for at least one week between 1954 and 2004. Follow-up was recently extended from 1986 to 2007 for mortality and included follow-up for cancer incidence from 1969-2005. Standardised mortality ratios (SMRs), standardised incidence ratios (SIRs) and their 95\% confidence intervals (CIs) will be calculated based on Canadian national reference rates.

Results The final cohort consisted of 28546 miners. The mean age of the miners at entry into the study was 28.8 years. Miners in the cohort had a mean cumulative exposure of 21.0 WLM over an average of 5.3 years of total exposure. Between 1954 and 2007, a total of 8572 deaths were observed, and of these 2809 were due to cancer, including 1246 lung cancer deaths. There were 4151 incident cancers, including 1285 lung cancers, observed.

Conclusions The Ontario uranium miner's cohort study continues to be a valuable source of assessing uranium miners risk of cancer mortality and incidence. 


\section{HEART RATE VARIABILITY IN PARTICLE EXPOSED TRAIN} DRIVERS IN THE STOCKHOLM SUBWAY

${ }^{1,2}$ Carolina Bigert, ${ }^{2}$ Magnus Alderling, ${ }^{3}$ Magnus Svartengren, ${ }^{1}$ Nils Plato, ${ }^{4}$ Martin Anderson, ${ }^{2}$ Andreas Wiklund, ${ }^{1,2}$ Per Gustavsson. ${ }^{1}$ Institute of Environmental Medicine, Karolinska Institutet, Stockholm, Sweden; ${ }^{2}$ Center for Occupational and Environmental Medicine, Stockholm County Council, Stockholm, Sweden; ${ }^{3}$ Department of Medical Sciences, Occupational and Environmental Medicine, Uppsala University, Uppsala, Sweden; ${ }^{4}$ Department of Clinical Physiology, Södersjukhuset, Stockholm, Sweden

\subsection{6/oemed-2014-102362.355}

Objectives Exposure to particulate matter in urban air is a recognised risk factor for cardiovascular disease, but little is known about possible effects from exposure to the high levels of metalrich particles prevailing in underground subway systems. This led us to investigate heart rate variability (HRV) in occupationally exposed subway drivers.

Method 29 train drivers (18 men and 11 women) in the Stockholm subway were investigated from November 2004 to March 2005. All were non-smokers in ages 25-50. Personal particle exposure levels were obtained in an occupational hygienic investigation (mean $\mathrm{PM}_{2.5} 19 \mu \mathrm{g} / \mathrm{m}^{3}$, DataRAM $33 \mu \mathrm{g} / \mathrm{m}^{3}$ ). We registered continuous ECG over $24 \mathrm{~h}$. The HRV measures obtained were LF, HF, LF/HF, HR and SDNN. The arithmetic mean (based on 5-minutes intervals) in the group was calculated for each measure and exposure situation, as well as the mean in group of the individual quotients between the exposure situations. One-sample t-tests were used to analyse whether the quotients differed from one.

Results The mean quotients between working in tunnel and working outside tunnel were significantly above one for LF ( $\mathrm{p}=$ $0.04)$ and significantly below one for HR $(\mathrm{p}=0.03)$ and SDNN $(\mathrm{p}=0.00)$. The quotients between total working-hours and leisure-hours were significantly above one for HR $(p=0.03)$ and significantly below one for SDNN ( $p=0.00)$.

Conclusions Overall, our results do not indicate any clinically significant effects on the cardiac autonomic function, as measured by HRV, for particle exposed subway drivers in Stockholm, even though there were some indications of a decrease in SDNN.

\section{WORKPLACE BULLYING AND POSTTRAUMATIC STRESS SYMPTOMS AMONG FAMILY PHYSICIANS IN LITHUANIA}

Vilija Malinauskiene, Lina Bernotaite. Lithuanian University of Health Sciences, Kaunas, Lithuania

\subsection{6/oemed-2014-102362.356}

Objectives The study investigated the associations between workplace bullying and posttraumatic stress symptoms as compared to and controlled for associations between the latter and other psychosocial stress factors at work and in everyday life, employing a representative sample of Lithuanian family physicians.

Method With a response rate $89.2 \%$, a total of 323 family physicians filled in anonymous questionnaire on workplace bullying, post traumatic symptomatology (IES-R), other psychosocial stressors at work and in everyday life, personal health resources (sense of coherence), behavioural characteristics and demographic variables. The statistical software SPSS 14.0 for Windows was used in the analysis. Associations were tested by way of multivariate logistic regression analysis.
Results A high prevalence of bullying was found among the family physicians in Lithuania, with 13\% experiencing severe workplace bullying and $17.3 \%$ more occasional incidents of bullying. The prevalence of posttraumatic stress symptoms was also high $(15.8 \%)$. The Odds ratio (OR) of severe bullying for posttraumatic stress after adjustment for age and gender was 8.05, 95\% confidence interval (CI) 3.80-17.04. In the fully adjusted model it increased to $13.88,95 \%$ CI $4.68-41.13$, indicating cumulative effects of all the investigated stressors.

Conclusions Workplace bullying is prevalent among Lithuanian family physicians, as is symptoms of posttraumatic distress. Strong associations between posttraumatic stress and exposure to severe bullying indicate that bullying is a significant source of mental health problems among physicians and more so than most other well known psychosocial stressors at work and in daily living.

\section{EPIDEMIOLOGY OF LOW BACK PAIN AMONG NURSES OF THE HOSPITAL OF SÉTIF (ALGERIA)}

1,2Ziadi Boukerma, ${ }^{1}$ Ahmed Lakhdar Behlouli, 'Meriem Reggad. 'Université Farhat Abbas Sétif 1, Sétif, Algeria; 'Laboratoire Santé Environnement Des Hauts Plateux Sétifiens, Sétif, Algeria

\subsection{6/oemed-2014-102362.357}

Objectives Identifying risk factors and quantification of the prevalence of low back pain among nurses and service agents working at the hospital in Sétif (Algeria).

Method A questionnaire containing 45 items was submitted by a physician to 450 people, of which only 300 people have agreed to meet. Responses have been verified by cross-checking with the information contained in the medical records of the occupational medicine service. Controlled data have been analysed by the chi-square test and multivariate logistic regression techniques using the "IBM SPPS 20" software.

Results The prevalence of LBP is $66.67 \%$, it is significantly higher between 30 and 49 years of age and between 2 and 5 years of seniority at the hospital. These low back pains have a gradual onset in $52.0 \%$ and radiates along the sciatic nerve in $62.0 \%$. The logistic regression analysis revealed the following associations: low back pain predicted by psychosocial factors (stress, sleep disturbance and fatigue late in the day), the usual working posture and frequent positioning of patients in bed. Regarding sleep disorders, we cannot say if they are a source or a consequence of low back pain even if they are strongly associated with this disease.

Conclusions Occupational factors that have a significant influence on the development of low back musculoskeletal disorders are not only mechanical and postural order but also organisational, social and psychological.

\section{RISK OF NON-HODGKIN LYMPHOMA IN HEALTH OCCUPATIONS}

${ }^{1}$ Michela Ursi, 'Marcello Noli, ${ }^{1}$ Federico Marras, 'Carlo Aresti, ${ }^{2}$ Andrea't Mannetje, ${ }^{1}$ Pierluigi Cocco. ' University of Cagliari, Monserrato, Cagliari, Italy; ${ }^{2}$ Massey University, Wellington, New Zealand

\subsection{6/oemed-2014-102362.358}

Objectives Several non-Hodgkin lymphoma (NHL) risk factors are typical features of health occupations. We investigated risk of NHL and its major subtype among health workers. 